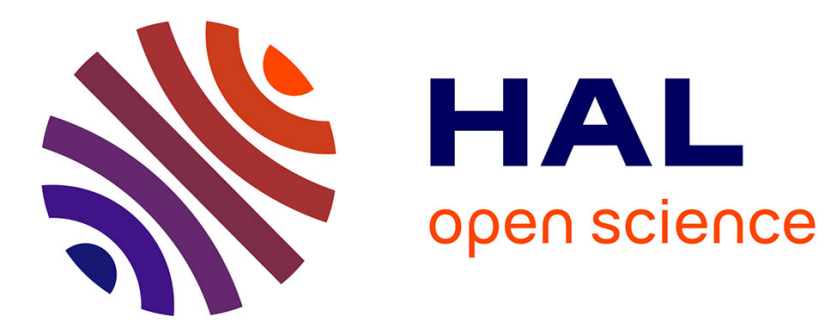

\title{
Geometric Interpretations of Conflict: A Viewpoint
}

Thomas Burger

\section{To cite this version:}

Thomas Burger. Geometric Interpretations of Conflict: A Viewpoint. BELIEF 2014: Belief Functions:

Theory and Applications, pp.412-421, 2014, 10.1007/978-3-319-11191-9_45 . hal-03212110

\section{HAL Id: hal-03212110 \\ https://hal.science/hal-03212110}

Submitted on 29 Apr 2021

HAL is a multi-disciplinary open access archive for the deposit and dissemination of scientific research documents, whether they are published or not. The documents may come from teaching and research institutions in France or abroad, or from public or private research centers.
L'archive ouverte pluridisciplinaire HAL, est destinée au dépôt et à la diffusion de documents scientifiques de niveau recherche, publiés ou non, émanant des établissements d'enseignement et de recherche français ou étrangers, des laboratoires publics ou privés. 


\title{
Geometric interpretations of conflict: a viewpoint
}

\author{
Thomas Burger * \\ iRTSV-BGE (Université Grenoble-Alpes, CNRS, CEA, INSERM), Grenoble, France \\ thomas.burger@cea.fr
}

\begin{abstract}
Recently, several works have focused on the study of conflict among belief functions with a geometrical approach. In such framework, a corner stone is to endow the set of belief functions with an appropriated metric, and to consider that distant belief functions are more conflicting than neighboring ones. This article discusses such approaches, caveats some of their difficulties and highlights ways to circumvent them.
\end{abstract}

Keywords: belief functions; conflict; distance

\section{Introduction}

Mass functions are rather simple objects from a mathematical point of view: distributions over a finite powerset which add up to one. However, their semantic is rich enough to be used in artificial intelligence to express the subjective opinion of an agent, in versatile frameworks such as imprecisely known statistics or censored data fusion. This difference between the simplicity of the mathematical object, and the subtlety of its interpretation at a high semantic level is probably the curse of belief function theory, as it prevents providing the scientist or engineer with a simple textbook or recipe, that is no longer put into question, on how to use belief functions to model a real world problem. For instance, after few decades of developments, the theory still lacks a unique and well established definition of conflicting belief functions. Since the seminal work of Dempster [8] where the mass in the emptyset has been used as a normalization factor in the orthogonal sum, the traditional conflict mass is regularly challenged with alternative definitions $[16,17,9]$.

This never-ending debate on the mathematical definition of conflict has been fed for few years now by a trend, which is discussed in the present paper. This trend is largely based on endowing the set of mass functions with a metric, and on characterizing the degree of conflict between two mass functions according to their pairwise distance. Historically, this direction was first initiated in 1996 by the work of George and Pal [10], which remains seldom cited (40 citations according to Google Scholar), while a few years latter (2001), Jousselme

\footnotetext{
* This work was supported by the ANR funding ANR-10-INBS-08, and the Prospectom project (Mastodons 2012 CNRS challenge). The author deeply thanks Sébastien Destercke and Didier Dubois whom discussions with fed this viewpoint.
} 
et al. [12] proposed their famous distance based on Jaccard's index. During the early 2000s, Cuzzolin developed his geometrical framework for belief functions, and Liu proposed to complement the conflict mass by the Chebyshev (or $L^{\infty}$ ) distance between pignistic transforms [16]. Jousselme's work being cited 400 times, Liu around 200 times and Cuzzolin three most cited papers [4-6] on the subject reaching more than 150 citations, we can fairly acknowledge that the interplay of these works, crystalized in the 2012 survey of Jousselme [14] (a presentation of which was given at BELIEF 2010, in Brest [13]) provided the technical ground, on which these new ways to define conflict has blossomed in the past years.

From that point on, numerous works have focused on providing a distance based definition of conflict. The objective of this article is not to review or to compare them. Rather, it is to go back to the foundation of this trend, and to address the interplays between the very mathematical notion of distance and the concept of conflict as acknowledged in belief function theory. After some definitions (Section 2), these interplays are discussed at a mathematical level (Section 3), and at a semantic one (Section 4).

\section{Definition}

Let us recall that a metric space $(E, d)$ is a set $E$ endowed with a distance $d$. A distance on $E$ is defined as:

1. An application from $E \times E$,

2. onto $\mathbb{R}_{+}$,

3. so that $\forall x, y \in E^{2}, d(x, y)=0 \Leftrightarrow x=y$,

4. $d(x, y)=d(y, x)$,

5. $\forall x, y, z \in E^{3}, d(x, z) \leq d(x, y)+d(y, z)$.

Usually, (2) is referred to as non-negativity, (3) as separability, (4) as symmetry and (5) as subadditivity, or triangle inequality. Although the term is not standard, let us refer to (1) as binarity for convenience. Beyond algebraic definitions, it is classically assumed in information-related sciences, that the semantic associated to a distance is that of dissimilarity: Greater the distance between two elements of $E$ the more dissimilar they are, and the smaller the distance, the more similar. In the particular case of belief function theory, $E$ is generally the set $\mathcal{M}(\Omega)$ of mass functions with frame $\Omega$. To simplify distance definitions, $\mathcal{M}(\Omega)$ is also often assumed to be a $(n-1)$ standard simplex in a $n$ dimensional normed vector space $V$ spanned by the $n$ possible focal elements that are canonically derived from working on $\Omega$ (see [6]). Thus, any norm defined on $V$ trivially leads to a distance on $\mathcal{M}(\Omega)$.

Let us now turn to the definition of conflicting belief functions: As explained in the introduction, the belief function community has not accepted a single mathematical definition for it, and scientists may derive a posteriori the mathematical definition which fit with the particular situation they face. It means that the definition is somehow clear at a "modeling" level, as everyone one can tell if different opinions are conflicting or not; however, this does not obviously 
translate into the formalism of belief functions. If we refine a bit the process, it appears that:

1. Several sources of information can agree or disagree on any phenomenon they have evidence on;

2. We call degree of conflict among the sources the level to which the sources disagree, yet no formal definition of such disagreement exists;

3. As the sources provide mass functions, a painless misnomer makes us talk about degree of conflict among the mass functions;

4. This degree of conflict is computed by applying various formulas to the mass functions.

Finally, the main difficulty is to define how agreement/disagreement of the sources translates into the result of a mathematical operation on mass functions, so that one can quantify it. During this "translation", distances are likely to be called for assistance. The reason is the following: In most applications, the level of conflict itself is of seldom interest, and the practitioner is more interested in the combination of the sources. This combination is somehow expressing a consensus among the sources. Naturally, one expects that consensus to be easier to find if the sources have similar opinions, while it is impossible to find it if the sources support completely opposite opinions. As the conflict is supposed to quantify this possibility to find a consensus, it makes intuitively sense to measure distances between the masses to combine.

\section{Mathematical discussion}

At this point, one needs to check that the idea behind conflict measurement is compliant with the mathematical definition of a distance. Regarding nonnegativity and symmetry, there should have very little problem: It is rather intuitive that the conflict is a non-negative quantity, and most of the works on the issue assume so. For instance, in [9], this assumption is explicitly included in our first axiom, referred to as Extreme conflict value; and in [17], one finds it in both the first axiom, simply called non-negativity, and in the fourth one, referred to as normalization, and which corresponds to our Extreme conflict value axiom. Similarly, it is also taken for granted that the conflict between $m_{1}$ and $m_{2}$ is the same as the one between $m_{2}$ and $m_{1}$. As such, both [17] and [9] propose an axiom named symmetry. However, the other properties (subadditivity, binarity and separability) do not directly fit, and they are separately addressed hereafter.

\subsection{The binarity issue}

First, let us consider binarity: So far, in most works devoted to conflict, it is assumed that $n>2$ belief functions can generate some conflict, and that this latter can be measured, which strongly goes against the binarity property. Thus, if distances are involved in the process of conflict quantitation, a rigorous $n$-ary extension is required. The most natural way to define such extension is to rely 
on hypervolumes, i.e. a distance in the binary case, an era in the ternary case, a volume in the quaternary case, and so on. Naturally, this raises lots of questions:

- How to deal with hypervolume computation in case of belief functions presented by collinear/coplanar vectors? The problem is that the volume spanned by a set of non full rank vectors is nil.

- How to compare the conflict arisen among a set of $n_{1}$ belief functions, and the conflict arisen among another set of $n_{2}$ belief functions? In fact, volumes of different dimensionalities are not comparable.

- Which type of hypervolume to consider? The smallest encompassing hypersphere, the simplex spanned by the masses, etc.?

However, it has two major advantages: First, hypervolume computation being insensitive to the order of the vertices under consideration, it naturally fits with the $n$-ary generalization of the symmetry property discussed above. Second, it can rely on matrix algebra as a robust mathematical background, where most of the results are well documented: Indeed, determinant or eigenvalue computations relates to the geometry of the column vectors of a matrix. So far, such an algebraic vision is seldom considered, and only weak justifications appear in [15].

\subsection{The separability issue}

Behind separability, one questions the equivalence between the following two assertions: "These masses are non-conflicting" and "These masses are equal". To answer, one should question the implication of the first assertion by the second, and the reverse implication; that is: Is it possible to find separable mass functions with fully agreeing sources? and, is it possible to find fully disagreeing sources with equal evidence?

Separable mass functions with agreeing sources? Let us remark that in belief function theory, focal elements are most of the time interpreted as epistemic sets [3], i.e. imprecise descriptions of the reality: one assumes the truth is one and only one element amongst those composing the set ${ }^{1}$. Under such view, it is possible to have sources of information having different descriptions with a non-null intersection that they can agree on. The direct conclusion is that, contrarily to distances, conflict is quantity that does not fit with the separability property. Let us illustrate that with some examples:

Example 1 (Everyday life situation with an epistemic view). In this example, we do not assume any model based on belief functions. We simply consider two distinct sources of information. The first one supports an interval $I_{1}$ (for a range of acceptable values, a set of possible options, or whatever its meaning), while the second source support another interval $I_{2}$ included in $I_{1}$. In such a case, both sources can agree on $I_{2}$ (there is no conflict), while their opinions differ.

\footnotetext{
${ }^{1}$ To the best of my knowledge, no work proposes a new distance-based definition of conflict while explicitely assuming an ontic view.
} 
Example 2 (Separable mass functions with fully agreeing sources). Assume that source $S_{1}$ provides a vacuous mass function $m_{1}$, and that source $S_{2}$ provides a non-vacuous consonant mass function $m_{2}$. One clearly has $m_{1} \neq m_{2}$, and thus $d\left(m_{1}, m_{2}\right)>0$ whatever $d$. However, $S_{1}$ and $S_{2}$ are not conflicting, as the evidence supported by $m_{1}$ is non-informative in an epistemic model.

It is also possible to define other similar examples by replacing $m_{2}$ by a consonant mass function having its focal elements included in those of $m_{1}$, while having $m_{1} \neq m_{2}$. Whatever the example, the underlying idea is that a part of the distance between two separate mass functions can arise from their respective level of imprecision, and that this difference does not necessarily imply any conflicting views. This idea is implemented in the Ignorance is bliss axiom from [9] and in the inclusion axiom from [17]. However, these axioms are not here taken as the initial assumption to build these counter-examples on; I only rely on the weaker assumption that belief functions theory assumes an epistemic model. On the contrary, these axioms can be seen as a consequence of the epistemic modeling.

Disagreeing sources with equal evidence? Let $m_{1}$ and $m_{2}$ be two mass functions such that $m_{1}=m_{2}$, and thus $d\left(m_{1}, m_{2}\right)=0$. Does this necessarily imply that the conflict between $m_{1}$ and $m_{2}$ is nil? This question should be sorted out with respect to the on-going discussions regarding the decomposition of conflict into its inner part and its outer part $[7,20]$. These works assume that the global conflict arising from $m_{1}$ and $m_{2}$ can be partly explained by the conflicts that are carried by $m_{1}$ on its own regardless $m_{2}$, and by $m_{2}$ on its own regardless $m_{1}$. As such the outer conflict is what remains from the global conflict once the inner conflicts have been subtracted. If $d\left(m_{1}, m_{2}\right)=0$ implies a null (global) conflict, one has to assume that inner conflict does not exist, which so far contradicts with several state-of-the-art articles. An alternative is to assume that a distance-based measure of conflict only accounts for outer conflict. However, this line is also source of difficulties, as so far, no exact decomposition of the conflict into its outer and inner parts holds.

\subsection{The subadditivity issue}

Let us finally and rapidly turn to subadditivity (or triangle ineqality). Is there any reason to consider that the conflict between $m_{1}$ and $m_{2}$, plus the conflict between $m_{2}$ and $m_{3}$ should be greater than the conflict between $m_{1}$ and $m_{3}$ ? The following example provides a situation where this property is not desirable.

Example 3. Assume that $m_{1}$ and $m_{3}$ are consonant mass functions bearing on different opinions, and each being different from the vacuous one. Naturally, they have a non-null conflict. Now, let us assume $m_{2}$ is the vacuous mass: it is absolutely non-conflicting with either $m_{1}$ or $m_{3}$ so that the sum of these two conflicts is nil, leading to a situation where the triangle inequality does not hold.

Of course, this example relies on Example 2 and assumes an epistemic model. However, it makes sense: in everyday life, it is possible to consider two extreme 
positions that are in total conflict while an in-between position is hardly conflicting with both, as it supports an acceptable solution for everyone. In such case, the triangle inequality does clearly not hold.

\subsection{To conclude on the distance properties}

Finally, amongst the five properties of metrics, two naturally fits to conflict definition (symmetry and non-negativity), one may accept some suitable generalization (binarity), and two are absolutely not compatible (subadditivity and separability) with an epistemic view of belief functions. At this point, we can argue that conflict and distances (or dissimilarities) are different notions which cannot be interchanged. Unfortunately, a lot of recent works assume it is possible to do so (see for example $[18,22,19,15]$ ), which is arguable. On the other hands, it is possible to agree on the mathematical differences between these notions, while proposing to build a sounded definition of conflict thanks to the involvement of an adapted metrics; most of the time, by coupling a distance measure to another index, such as in $[16,17]$. This second approach authorizes much more rigorous works. However, one needs to remain cautious regarding the semantic of such aggregation. For instance, what is the meaning of a multi-dimensional conflict [16], or of multiplying a distance by an inclusion measure [17]? This question is partly addressed in the next section.

\section{Semantic discussion}

Now, I leave the axiomatic discussion to question how conflict and distance may interplay at a semantic level, i.e. at the moment when the practitioner involves belief functions to model a particular real life phenomenon. I will first push forward the line initiated in Section 3, by questioning the semantic of an aggregation function used to build a measure of conflict on the basis of, among others, a distance measure. Second, I will discuss the semantic of $V$, the normed vector space derived from $\mathcal{M}(\Omega)$. Finally, I will question the definition of a consensus between several sources when the conflict is geometrically defined.

\subsection{Between similarity and agreement}

Let us assume that a measure of conflict is defined thanks to an aggregation function which takes as input, among others, a distance measure. Let $m_{1}$ and $m_{2}$ be two mass functions, and $\mathbf{C}$ a conflict measure reading:

$$
\mathbf{C}\left(m_{1}, m_{2}\right)=f\left(d\left(m_{1}, m_{2}\right), \mathbf{V}_{1}\left(m_{1}, m_{2}\right), \ldots, \mathbf{V}_{n}\left(m_{1}, m_{2}\right)\right)
$$

where $d$ is a distance over $\mathcal{M}(\Omega), f$ an aggregation function, and $\mathbf{V}_{1}, \ldots, \mathbf{V}_{n}$, $n \geq 1$ is a set of variables meaningfully describing some properties judged as interesting to quantify conflict. For instance: 
- in [16], $d$ is the Chebishev distance, $f$ is a concatenation operator that builds a vector on the basis of a list of its coordinates, $n=1$, and $\mathbf{V}_{1}\left(m_{1}, m_{2}\right)$ is the conflict mass.

- in [17], $d$ is the Jousselme distance, $f$ is a product, $n=1$ and $\mathbf{V}_{1}\left(m_{1}, m_{2}\right)$ is an inclusion measure.

- one could also assume that a distance is involved to measure the outer conflict, and that this latter is complemented with another measure accounting for inner conflicts.

All these works assume that similarity and agreement are different notions, which is so far supported by Section 3. However, they make an additional assumption, which is that a distance can be combined with a finite set of other values, namely $\mathbf{V}_{1}\left(m_{1}, m_{2}\right), \ldots, \mathbf{V}_{n}\left(m_{1}, m_{2}\right)$ so that the resulting combination is a conflict measure that fairly describes the level of disagreement of the sources. So far, I do not have any support for this assumption, nor any argument against it. However, the assumption is often associated to a constructive approach I find prone to discussion: Generally, a measure of conflict of the form of $\mathbf{C}$ is built iteratively:

1. One starts from a first definition of conflict (either $\mathbf{V}_{1}\left(m_{1}, m_{2}\right)$ or $d\left(m_{1}, m_{2}\right)$ in the aforementioned examples);

2. One finds a counter-example on which this definition does not entirely capture what one expects as conflict;

3. One proposes a correction, generally by incorporating another variable, say $\mathbf{V}_{i}\left(m_{1}, m_{2}\right)$. At this point, if the resulting definition of conflict is not sounded, one goes back to the previous step.

During this process, one never has the confirmation that the collection $\left\{d\left(m_{1}, m_{2}\right)\right.$, $\left.\mathbf{V}_{1}\left(m_{1}, m_{2}\right), \ldots, \mathbf{V}_{n}\left(m_{1}, m_{2}\right)\right\}$ is complete: As long as no example puts it back into question, the definition is accepted; while, on the contrary, one should accept it only after proving its completeness. Practically, when discussing the separability issue in Section 3, I pointed out that some distance could arise from the different levels of imprecision between $m_{1}$ and $m_{2}$, without implying any conflict. A similar conclusion was drawn in [17], so that the proposed measure complements a distance by an inclusion measure. However, there is no evidence supporting that the resulting measure of conflict is complete.

\subsection{Sensitivity to permutations}

The second issue to focus on is best illustrated by an example:

Example 4. Let $v_{1}=(1,0,0)^{\top}$ and $v_{2}=(0,1,0)^{\top}$ be two vectors of $\mathbb{R}^{3}$, and let $\sigma \in S_{3}$ be a permutation. As any permutation of the coordinates of a vector can be decomposed into a succession of cycles (which intuitively corresponds to rotations of the basis of $\mathbb{R}^{3}$ ), it is rather intuitive that most of the canonical distances (such as Minkowski distances) on $\mathbb{R}^{3}$ are insensitive to permutations: Practically, if $d$ is such a distance, then $d\left(v_{1}, v_{2}\right)=d\left(\sigma\left(v_{1}\right), \sigma\left(v_{2}\right)\right), \forall \sigma \in S_{3}$. 
However, if $v_{1}$ and $v_{2}$ are intepreted as mass functions (let us name them $m_{1}$ and $m_{2}$ ) over a binary frame the powerset of which reads $\left\{\left\{\omega_{1}\right\},\left\{\omega_{2}\right\},\left\{\omega_{1}, \omega_{2}\right\}\right\}$, things become different: $m_{1}$ and $m_{2}$ are naturally conflicting as they respectively fully support $\left\{\omega_{1}\right\}$ and $\left\{\omega_{2}\right\}$, however, if $\sigma$ is the cycle $(2,3)$, then $\sigma\left(m_{1}\right)=$ $(1,0,0)^{\top}$ and $\sigma\left(m_{2}\right)=(0,0,1)^{\top}$ are not conflicting, as $\sigma\left(m_{2}\right)$ is vacuous.

The above example illustrates that, contrarily to a classical vector space such as $\mathbb{R}^{n}$, where the order of the coordinates of a vector are seldom important, $\mathcal{M}(\Omega)$ is rather sensitive to permutations. Naturally, this example also relies on the same tricks as Examples 2 and 3: one involves a vacuous mass function and one assumes it is not conflicting with any other consonant mass. However, this is mainly to keep the example both simple and catchy. What really matters here is much more general: as the semantic of each coordinate of a vector is not the same (some correspond to singleton focal elements, while others do not, respectively encoding precise knowledge, or not), any permutation of the vector space strongly modifies the semantic of the mass functions. However, several classical distances are unchanged by permutations. Thus, the sensitivity to permutation must be explicitly accounted for, if one expects a distance to be involved in a conflict definition. Jousselme's distance based on Jaccard's index, as well as other entropy-related distances, partially do, yet in different manners. However, other distances defined on $\mathcal{M}(\Omega)$ may not (including most of the canonical distances on a vector space).

\subsection{Geometric definition of consensus}

When combining belief functions, the resulting mass is supposed to reflect a consensus among the original sources. This consensus aims at being as compatible as possible with the pieces of evidences provided by the sources, while being specific enough (a vacuous mass is not a relevant consensus). Let us explore the consequences of that, in the case where conflict is reflected by distances. If the degree of disagreement between the sources if fairly captured by a distance measure, the consensus mass function is expected to have a minimal distance to all the original pieces of evidence. From a geometrical viewpoint, such a mass function is defined by the barycenter of the sources to combine. Thus, the merging of the pieces of information should not be conducted according to Dempster's rule, but according to a convex combination. Although in plain contradiction with the mainframe of belief function theory, the idea that a consensus is well-defined by a barycenter has already showed up in the literature: In [21], Assumption 1.1. reads that the pignistic transform should be invariant with respect to convex combination rather than with respect to Dempster's rule, such as advocated in [2]. In a slightly less related way, the pignistic transform is easily interpreted in terms of linear combination: It provides the barycenter of dominating probabilities [1], which can be interpreted as a geometric consensus between all the compliant probability distributions. However, by now, Dempster's rule is well established (it is seldom put into questions, such as in [11]), and such a change would have major impacts on the theory (far beyond the simple definition of conflict) and its consequences should be globally considered. 


\section{Conclusion}

This article discusses the recent trend which focuses on using metrics to quantify the degree of conflict between belief functions. As I am sure it appeared through this discussion, I am not a supporter of this trend, and my views remains coherent with a previous article [9] I was a co-author of, where one has defined various axioms that we believe a sounded measure of conflict should meet. However, I understand that belief functions have rich and multiple semantics that may differ, and among which none is better than the others. As such, in this viewpoint, I considered the question through some constructive Cartesian skepticism: I tried to push as far as possible the line of mixing distances and conflict, with the hope that it would raise interesting questions.

As a result of the axiomatic discussion conducted in Section 3, it appears that the separability and subadditivity properties of distances contradict what can be expected from a conflict measure. It clearly indicates that, at a low mathematical level, the notions of conflict and of distance cannot be interchanged. However, at this point, it is impossible to reject another trend, which consists in elaborating a measure of conflict thanks to an aggregation function which takes into account, among others, a distance. This is why, in Section 4, I discuss the differences between distances and conflict at a semantic level, through different angles.

Finally, it appears that even if no blatant contradiction appears at this semantic level, several major issues remain, if one expects to build a complete vision-based definition of conflict:

1. Such as discussed in Section 3.1, a suitable $n$-ary $(n>2)$ generalization of the distance is necessary. Moreover, this generalization should be insensitive to permutations, to fit with the symmetry property;

2. One only considers outer conflict (thus, a suitable separation of inner and outer conflict is mandatory), such as advocated in Section 3.2;

3. An exhaustive definition of all the differences between agreement and similarity must be given, so that the distance is aggregated to another set of measures accounting for these differences, such as it is outlined in Section 3.2 and detailed in Section 4.1;

4. The distance must be defined so that it has a semantic robust to permutations of the focal elements, in order to avoid situation such as illustrated in the Example 4 (Section 4.2);

5. The combination rule must be adapted to fit a barycentric vision of the consensus, such as described in Section 4.3.

Finally, most of these issues are real locks that can only be addressed in dedicated works. According to the amount of works focused on the definition of a geometric and distance-based vision of conflict, the subject is definitely an interesting question for the belief function community. However, its complete and rigorous definition still requires several issues (at least, the five aforementioned) to be solved beforehand. Should these issues be sorted out, it would be interesting to confront a distance-based conflict to pre-existing definitions. 


\section{References}

1. Burger, T., Cuzzolin, F.: The barycenters of the k-additive dominating belief functions \& the pignistic k-additive belief functions. In: Workshop on the Theory of Belief Functions (2010)

2. Cobb, B.R., Shenoy, P.P.: On the plausibility transformation method for translating belief function models to probability models. Int. J. Approx. Reasoning 41(3), 314-330 (2006)

3. Couso, I., Dubois, D.: Statistical reasoning with set-valued information: Ontic vs. epistemic views. Inter. J. Approx. Reasoning (2013)

4. Cuzzolin, F.: Geometry of dempster's rule of combination. Systems, Man, \& Cyb. (B), IEEE Trans. 34(2), 961-977 (2004)

5. Cuzzolin, F.: Two new bayesian approximations of belief functions based on convex geometry. Systems, Man, \& Cyb. (B), IEEE Trans. 37(4), 993-1008 (2007)

6. Cuzzolin, F.: A geometric approach to the theory of evidence. Systems, Man, and Cyb. (C), IEEE Trans. 38(4), 522-534 (2008)

7. Daniel, M.: Conflicts within and between belief functions. In: IPMU. pp. 696-705 (2010)

8. Dempster, A.: Upper and lower probabilities induced by a multivalued mapping. Annals of Mathematical Statistics 38, 325-339 (1967)

9. Destercke, S., Burger, T.: Toward an axiomatic definition of conflict between belief functions. Systems, Man, \& Cyb. (B), IEEE Trans. 43(2), 585-596 (2013)

10. Geoarge, T., Pal, N.R.: Quantification of conflict in dempster-shafer framework: A new approach. Int. J. Gen. Sys. 24(4), 407-423 (1996)

11. Jøsang, A., Pope, S.: Dempsters rule as seen by little colored balls. Computational Intelligence 28(4), 453-474 (2012)

12. Jousselme, A.L., Grenier, D., loi Boss: A new distance between two bodies of evidence. Information Fusion 2(2), 91 - 101 (2001)

13. Jousselme, A.L., Maupin, P.: On some properties of distances in evidence theory. BELIEF, Brest, France (2010)

14. Jousselme, A.L., Maupin, P.: Distances in evidence theory: Comprehensive survey and generalizations. Int. J. Approx. Reasoning 53(2), 118 - 145 (2012)

15. Ke, X., Ma, L., Wang, Y.: A dissimilarity measure based on singular value and its application in incremental discounting. In: Information Fusion. pp. 1391-1397. IEEE (2013)

16. Liu, W.: Analyzing the degree of conflict among belief functions. Artificial Intelligence 170(11), $909-924$ (2006)

17. Martin, A.: About conflict in the theory of belief functions. In: Belief Functions: Theory and Applications, pp. 161-168. Springer (2012)

18. Martin, A., Jousselme, A.L., Osswald, C.: Conflict measure for the discounting operation on belief functions. In: Information Fusion, 11th International Conference on. pp. 1-8 (2008)

19. Sarabi-Jamab, A., Araabi, B.N., Augustin, T.: Information-based dissimilarity assessment in dempster-shafer theory. Knowledge-Based Sys. 54, $114-127$ (2013)

20. Schubert, J.: The internal conflict of a belief function. In: Belief Functions: Theory and Applications, LNAI, vol. 164, pp. 169-177. Springer Berlin Heidelberg (2012)

21. Smets, P.: Decision making in the tbm: the necessity of the pignistic transformation. Int. J. Approx. Reasoning 38(2), 133-147 (2005)

22. Yang, J., Bai, B., Jiang, X., Liu, S., Huang, H.Z., He, L.P.: A novel method for measuring the dissimilarity degree between two pieces of evidence in dempstershafer evidence theory. In: QR2MSE. pp. 894-899 (2013) 